\title{
Revisiting Chemistry for Surgeons
}

\author{
J. S. Rajkumar ${ }^{1} \cdot$ Chintamani $^{1} \cdot$ Deepa Ganesh ${ }^{1}$
}

Published online: 6 July 2016

(C) Association of Surgeons of India 2016

The readers would recall an earlier editorial on "Basic Physics revisited for surgeons"; this write-up is essentially an extension of the same theme, the "science of surgery" or "science in surgery." Although one does not see it at the surface level, a surgeon, too, has got to consider and constantly evaluate the chemical equations that are occurring inside the patients. For example, something as simple as suturing a laceration in an emergency room should make the surgeon consider the chemistry of blood coagulation within the patient as well as the neuro chemistry of the process of pain. Not only this, if the patient is on a drug like aspirin, one will have to consider its effects on both these processes. For convenience, the write-up has been divided into two sections, one that deals with chemical equations in every day clinical situations and the second half of the article that concentrates on chemistry and biotechnology in the surgical industry, again, of relevance to the practicing surgeon. Of course, there are areas where the physics and chemistry would overlap, for example, thermo chemical equations, but a sincere effort has been made to remain outside the realm of the esoteric.

\section{Section A: Chemistry in Pre-operative, Per-operative, and Postoperative Situations}

A number of examples abound in literature about the importance of chemistry and chemical equations, but a few examples are highlighted and elaborated upon in order to kindle the avid young surgeons' thirst for knowledge.

Chintamani

drchintamani7@gmail.com

1 VMMC, Safdarjang Hospital New Delhi, New Delhi, India
1) Post operative oliguria: Sometimes, in the postoperative period, the patient shows a sharp fall in urinary output. There are many causes for such a phenomenon, but chief in the surgical resident's mind should be two, namely hypovolemic oliguria, due to under perfusion of the glomeruli, and the oliguria of acute tubular necrosis, in which the glomeruli are perfused adequately, but absorption does not take place at the level of the proximal convoluted tubules (Figs. 1 and 2). This problem is often resolved by a fluid challenge namely running in about half a liter of saline or ringer lactate (RL) within half to $1 \mathrm{~h}$ and observing if the urine output increases. Such an increase indicates that further perfusion of the glomeruli is warranted, and the clinical answer in such a situation would be to increase the intravenous fluids. However, in some situations, the extra fluid load might well be deadly to an already compromised or sick patient.

In this scenario, the urinary sodium test is a very useful one. It is based on the fact that a lower than normal urine sodium level is seen in dehydration and fluid loss-induced oliguria. A test that would cost less than Rs. 100 (approximately \$2) will help us accurately identify the actual cause of oliguria in the critical phase of postoperative care.

2) Anastomotic leak:

Another important critical care situation is one of postoperative observation of an anastomosis especially a colorectal anastomosis and we all are aware that the leak can be a disaster. Although, technically, this could be reflected in any leak, a number of studies have indicated that in an acute colorectal leak, a fairly consistent marker is a rise spike in serum sodium and a sharp decline in serum potassium, accompanied by a high total leucocyte counts. The biochemical basis of this finding is that any leak, involving a spillage of luminal contents into the 


\section{ACUTE TUBULAR NECROSIS}

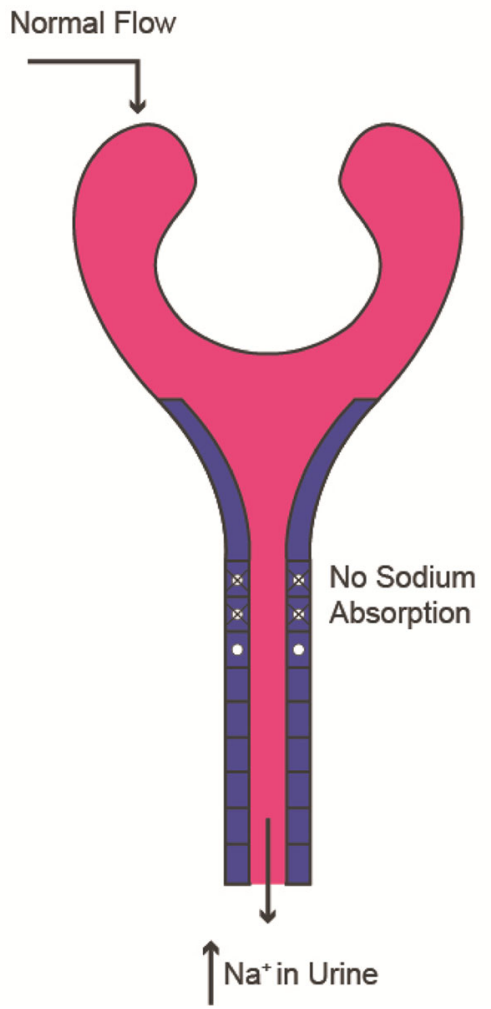

Fig. 1 Acute tubular necrosis

peritoneal cavity, will trigger off the stress response and will be accompanied by a flush of steroids into the circulation. Both glucocorticoids and mineralocorticoids have a common property of holding back sodium and pushing out potassium in response to stress. It is this flush of steroids that is reflected as an acute hypernatremia and acute hypokalemia in a postoperative patient. Combining this with a spike in total count and highly sensitive C reactive protein increases the sensitivity of this trio to detect this postoperative abdominal catastrophe, when still can be contained and redressed.

3) The Quantiferon test: Chemicals are often released by various cellular components in the blood, in response to perceived stress or foreign assault. This chemical release can be quantified and often allows us to guesstimate the nature of the offending agent. For example, we know that tubercle bacilli are approached for destruction by macrophages. On contact with the mycobacterium tuberculosis, the macrophages release a specific type of interferon, called gamma interferon. Thus, when there is doubt about the presence of tuberculosis, one can use a quantitative analysis of the amount of specific gamma interferon released by the macrophages, which will give one a fairly good idea of the possibility of tuberculosis. Although the TB gold or Quantiferon test is fraught with false positives and false
HYPOVOLEMIA

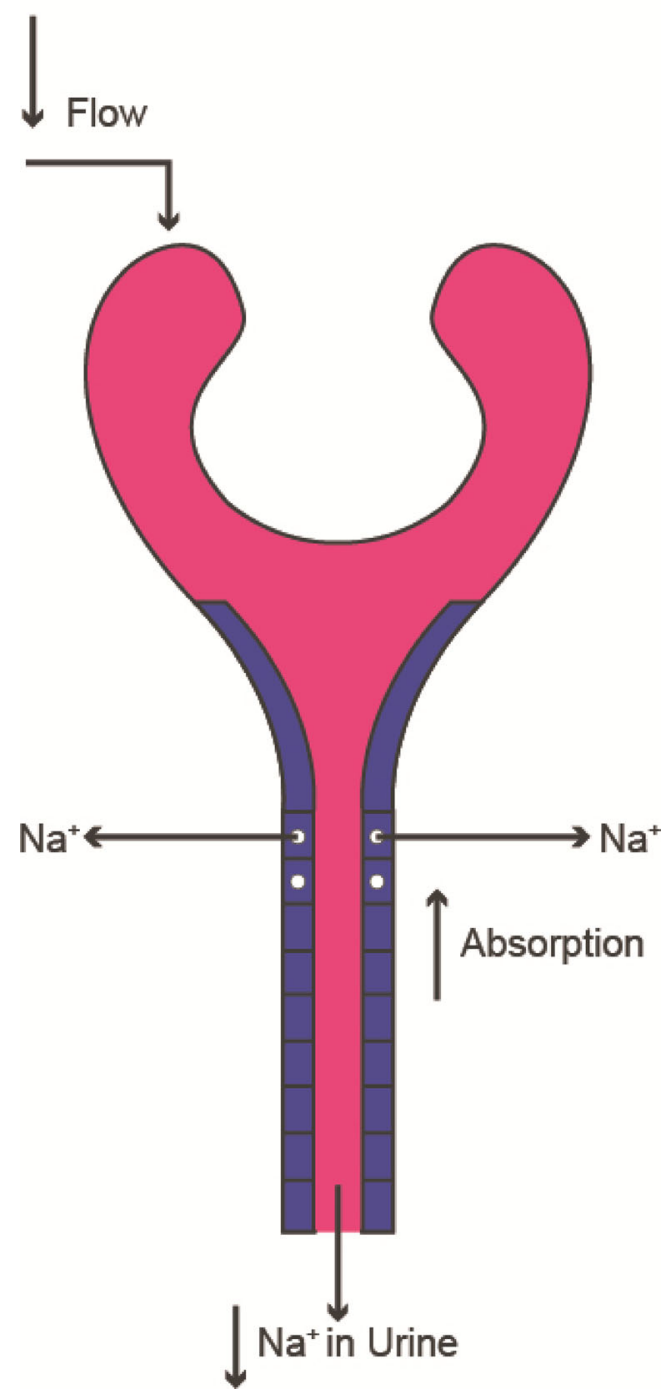

Fig. 2 Hypovolemic oliguria

negatives, it is still one of the few tests available for clinicians to make the diagnosis of tuberculosis.

4) Pheochromocytoma: Release of certain chemicals like epinephrine and nor-epinephrine from the adrenal medulla is associated with widespread hemodynamic changes during the surgical procedures. For example, patients with pheochromocytoma have a high circulating volume of catecholamines. These vasopressors act on the peripheral vasculature and keep up the sympathetic tone of the vessels. The result of these chemicals is that the plasma volume is effectively contracted for these patients. If these patients are operated upon, and the tumor is removed, then the level of circulating catecholamines drops drastically. This results in a sudden stoppage of stimulation of the peripheral vascular tone, and the contracted blood volume compartment suddenly expands. 
This massive expansion of the blood volume compartment causes a drastic drop in arterial pressures, and this intraoperative cataclysm may then result in a stroke, myocardial infarction, or renal failure. In order to prevent this event from occurring, the surgeon should consciously commence combined alpha, beta blockade of the receptors for a couple of weeks before surgery and should proceed to load up the patients circulating blood volume during the operation maximally. Thus, when the tumor is removed, and the catecholamine levels drop, the extra volume that is present will occupy the expanded circulatory compartment. It is also useful to have intravenous vasopressors to block the rid of the fall of the pressure, as also intravenous hypotensive agents to offset the rise in blood pressure that is consequent in the handling of tumor; thus, the knowledge of chemical release from the adrenals is of paramount importance in preventing and tackling an intra-operative crisis in pheochromocytoma surgery.

5) Neuroendocrine tumors and the DOPA reaction:

Another chemical reaction that is useful for all surgeons to know is the dopa reaction. Phenylalanine, a basic amino acid, gets hydroxylated to become hydroxyl phenylalanine, or tyrosine. Tyrosine gets further hydroxylated to become dihydroxy phenylalanine, or DOPA (see Fig. 3). This dopa reaction is a very specific for all neuro endocrine cells in the body, which originate from the neural crest, in the fifth week of intrauterine life. Although they populate the entire length and width of the body, from the hypothalamus to the Kulchitsky cells of the bronchus and the cells of the islets of Langerhans in the pancreas, they all retain their common genetic fingerprint in the form of the chemical equation described previously. Thus, one of the ways of diagnosing a neuroendocrine origin for a tumor is to check a dopa reaction.

As a further option of the same, the release of the wide range of chemicals, either glucagon, insulin, vaso-active intestinal peptide, or serotonin from the specialized input cells begins with the DOPA reaction and goes through a metabolite called chromogranin alpha. If a patient complains, for example, of persistent severe watery diarrhea, along with the usual stool examination and colonoscopy, if a clinician had the test of chromogranin alpha, elevation of the same would strongly indicate a possible neuroendocrine tumor releasing either vaso-active intestinal

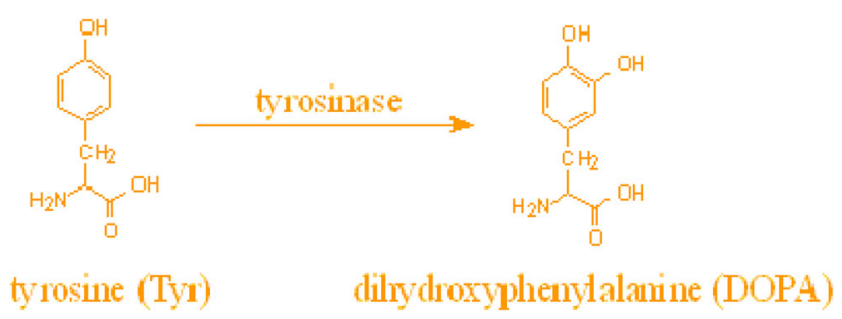

Fig. 3 DOPA reaction peptide (VIPoma) or, sometimes, a carcinoid (releasing serotonin). Thereafter, the more expensive specific chemicals can be qualitatively and qualitatively assessed in the serum and help with the diagnosis of this peculiar spectrum of diseases.

6) Dumping syndrome: Raoult's law is a chemical equation in relation to solutes dissolved in solutions (see Fig. 4) [1]. In essence, it states that the pressure of solutes excreted in a solution, is the sum total of the pressure exerted by the different solutes mixed in the same. Although a not frequently discussed law, it does play a role in the pathogenesis and treatment of the dumping syndrome [2].

This refers to the postprandial giddiness and weakness that follows a meal in a patient with a gastro-jejunostomy. Earlier classified as "early dumping," it is basically a sharp fall in blood pressure that happens postcibal in patients with gastro-jejunostomies. A number of studies have shown that this was due to an outpouring of fluid into the proximal jejunum. The actual reason for the fluid accumulation in the jejunal lumen is the presence of solutes, and its mixed carbohydrate solute will carry the maximum osmotic pressure and will pull in a correspondingly large volume of fluid with it. This loss of fluid from the intravascular compartment has the same hemodynamic effect as a large bleeding, causing hypotension and tachycardia and forcing the patient to lie down in the supine position for some time until equalization of pressures has occurred. Having understood that multiple miscible carbohydrate solutes exert the maximum osmotic pressure because of Raoult's law, one can easily solve the problem by decreasing the solutes osmotic pressure in the lumen of the jejunum. This can be affected by requesting the patient to take a high protein, low carbohydrate, low solute meal, and this is usually found to be therapeutic for early dumping after gastro-jejunostomy.

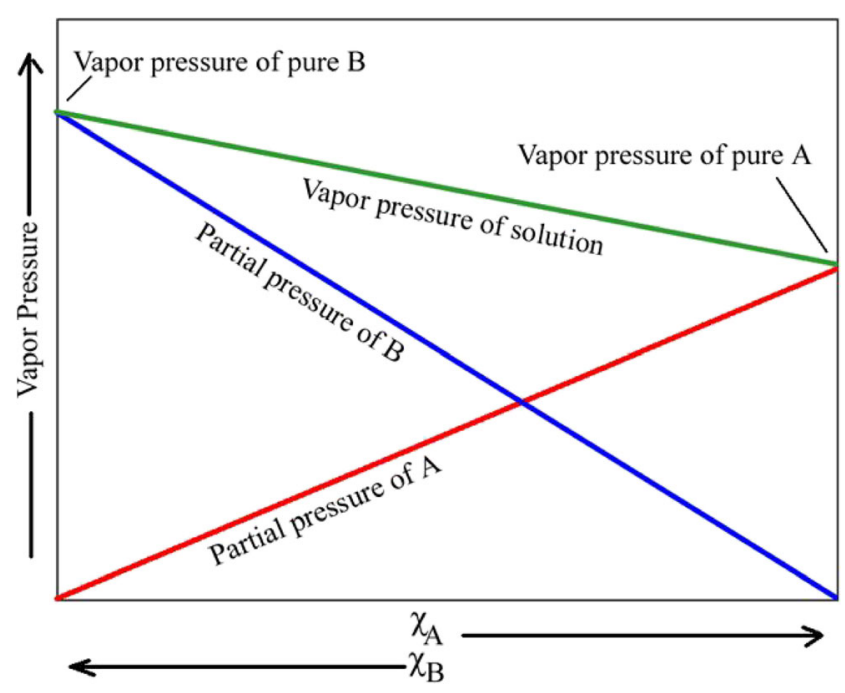

Fig. 4 Raoult's law-ideal solution 
The same therapy also works, paradoxically, for the late dumping, because it is a high carbohydrate load that triggers off a high insulin response, precipitating hypoglycemia towards postprandially in patients with gastro-jejunostomies. By restricting solute and carbohydrate load, and decreasing the immiscible protein intake, one also prevents the 2 -h rise of insulin, and the postprandial hypoglycemia of the late dump.

7) The Gault-Cockroft equation: This chemical equation is of importance to surgeons in estimating a patient's glomerular filtration rate. The later is the most commonly used index to represent the renal function. It equates to the amount of fluid passing through the glomerulus, namely undergoing filtration. Technically speaking, the best way to identify a patient's glomerular filtration rate, and consequently his renal function, is to perform an inulin clearance, or radioactive isotope study, but these are both expensive, time consuming, and cumbersome.

Gault and Cockroft came up with a much simpler way of evaluating the renal function, which was known as the creatinine clearance. Creatinine clearance refers to the amount of plasma that is cleared of creatinine in a minute [3]. This is done by collecting the urine volume over $24 \mathrm{~h}$ and checking the urine creatinine and the plasma creatinine. The Gault-Cockroft formula is $\mathrm{C} / \mathrm{cr}=\mathrm{Ucr} \times \mathrm{V} / \mathrm{Pcr}$.

$$
\mathrm{CrCl}=\frac{(140 \text {-age }) \times \text { weight }(\mathrm{kg})}{\text { SerumCr }(\mathrm{mg} / \mathrm{dl}) \times 72}
$$

This creatinine clearance by the $\mathrm{CG}$ formula approximates to the GFR, but not exactly. The reason is that a small amount of creatinine is actually actively secreted into the proximal convoluted tubules by the peritubular capillaries. This accounts for the creatinine clearance being always an overestimate of renal function. Another formula, the MDRD, has proved to be a little more accurate than the time-tested Gault-Cockroft equation.

These formulae need to be especially kept in mind when evaluating the risk factors for postoperative renal insufficiency in a patient on multiple drugs and needing to undergo major surgery. Especially in the surgery of aortic aneurysm, with possible renal arterial cross clamping, the pre-existing renal status will often determine postoperative morbidity and sometimes mortality. Although we have our intensive care physicians to apply these complex formulae, it behooves the sharp surgeon to at least be aware of these!

The above are some examples of the judicious use of chemistry and chemicals in surgical practice. As mentioned earlier, the literature abounds with these but this is a clarion call to the young and budding surgeons to be constantly aware of chemistry as the basis of life.

The second section deals with the chemistry of implants, meshes, and hemostatic agents, to whet the appetite of all and especially the young surgeons.

\section{Meshes}

Hernias remain the commonest in general surgical practice, and vast clouds of controversy often surround the fabric materials of the meshes deployed to prevent recurrence of the hernia. Ever since the seminal work by Lichtenstein et al. [4], Gilbert [5], Rutkow [6], and Robbins, the effectiveness of meshes in preventing recurrence has gotten established. However, which mesh would be worth using? At this point of time, there are a confusingly large number of options available to the fairly well-read surgeon, as an attempt would be made to present the salient details of the various suitors!

Regular Polypropylene Mesh This is a regular mesh, fobbed by polypropylene polymerization, and is fairly good for most open inguinal, abdominal, and femoral hernias. It is strong, inexpensive, and is acceptable as long as it does not get infected (Fig. 5).

One of the areas in which chemistry plays an important role, is in the modification of meshes. The earlier meshes were simple polymerized, stretched, and cut fibers of polypropylene. Essentially, it was a net of graded polypropylene [7] (Marlex). The netted fraded polymerized fibers carried a tiny weight, and consequently the initial polypropylene meshes were all high molecular weight (HMW) meshes, more than $80 \mathrm{Da}$. Later with realization that many of the heavy duty meshes caused nagging pain and ache at the site of their application, and the knowledge that the tensile strength provided by the meshes was much more than that required for abdominal wall strength (a full sneeze or cough heals a maximum pressure of $150-200 \mathrm{mmHg}$, whereas the pressure held by a polypropylene mesh will be up to $700 \mathrm{mmHg}$ ), a move was made by the industry to decrease the amount of polypropylene and to reduce the molecular weight in the same. This resulted in the newer low-molecular weight meshes (Figs. 6 and 7). The principle of these meshes was to get two chemically different substances together. Also, if one substance were to undergo spontaneous chemical dissolution,

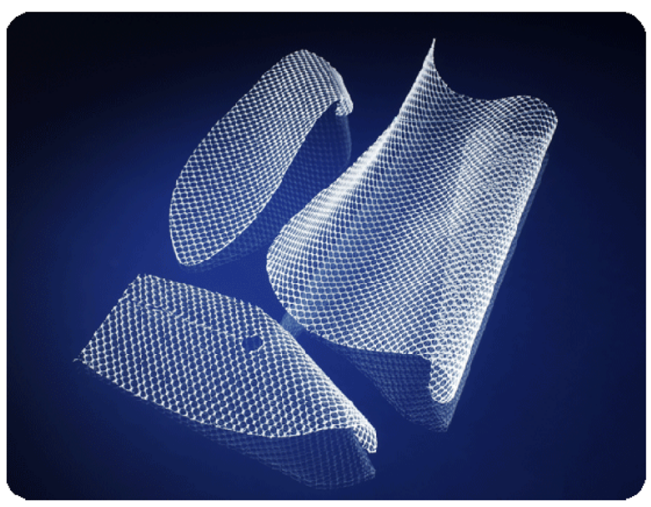

Fig. 5 Polypropylene mesh 


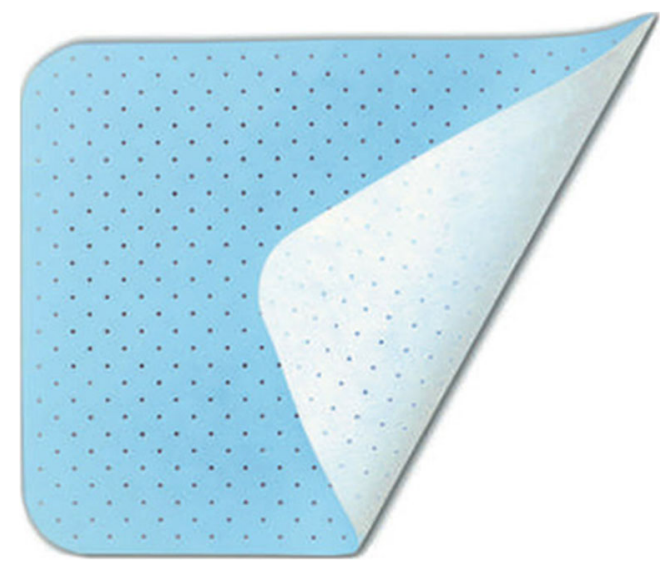

Fig. 6 Pro-Visc mesh

then the net weight of the mesh would only be contributed by the permanent substances. The composite meshes thus made their appearance too. In these, polypropylene is utilized to $50 \%$ of the weight of the mesh, and the remaining $50 \%$ is made up with polyglactin 910 (vicryl) or polyglecaprone (monocryl). Combining these two chemicals made a very big difference in hernia mesh industry and the lightweight and composite meshes have indeed started replacing the heavy molecular weight meshes of pure polypropylene.

The polymerization and fibrillary component of the chemical polypropylene is also of great importance in surgery. If one compares this chemical with another chemical widely used for hernias initially, namely PTFE or polytetrafluroethylene, one would find that the microscopic nature of the polytetrafluroethylene yields tiny "pores" of 1 to 2 microns only. The pore diameter between most of the polypropylene used currently is 7 to 10 microns, or even more. This has a huge impact on the persistence of infection.

Most bacteria that infect meshes have a diameter of 1 to 2 microns. They are able to slip through the pores of either of the meshes or stay in hiding. The average white blood cell has 7- to 8-micron diameter. It is therefore unable to enter the pores between the poly-tetra-fluroethylene, and clear out the bacteria, whereas it is comfortably able to do so in the larger pores found in the polypropylene. The net result of this is that

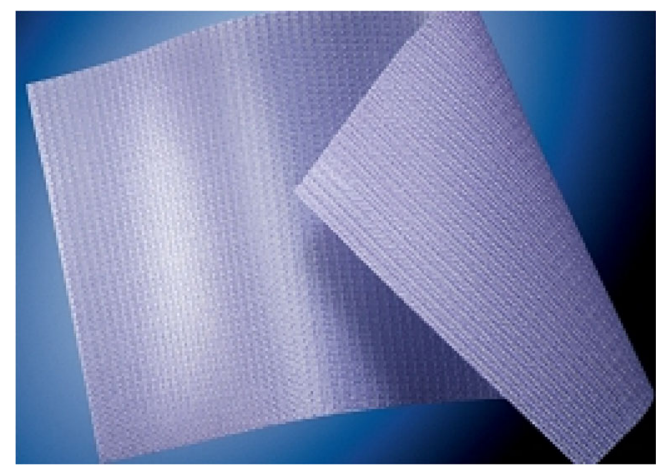

Fig 7 Sepra mesh one very rarely needs to remove a polypropylene mesh that is inserted in an open hernia procedure. One just needs to wash it out and clean and dress, while the white cells of the granulation tissue will do the job of cleaning out all the bacteria from the mesh. On the other hand, virtually all PTFE meshes that get infected have to be removed and hence the displacement of the PTF mesh by the former in common surgical practice.

Intra-abdominal Meshes With the era of laparoscopic ventral hernia repairs came the need to keep the small bowels away from the inner aspect of the mesh. The greatest worry is adhesion to the small bowel mesh, with resultant fistulization. Therefore, any mesh put internally to bridge ventral hernia defect needed to be coated with some chemical that would dissuade the small bowel from adhering to it. Several chemicals have been used to coat the mesh, with variant degrees of success. One such chemical is omega 3 fatty acid, namely, eicosapentaenoic acid (EPA) or docosahexaenoic acid (DHA), when coated on to the polypropylene served to repel the small bowel from attaching to it; another material that has been used is oxidized regenerative cellulose or ORC. This is commonly used as interceed, an adhesion barrier, and an absorbable hemostatic, which by dint of its high negative charge, serves to repel small bowel. Another polymer that has been used is polyurethane, an inert polymer with negative charge, used as an adhesion barrier. Although no single adhesion barrier has proved to be better for this distressing event of small bowel adhesion, by and large, a wide variety of chemicals described as above have manifested varying degrees of success in the ongoing battle against adhesions to meshes.

Styptics Styptics or hemostatics are locally acting chemicals that can accelerate the process of coagulation, stopping bleeding at the surgical site. As a source of extrinsic tissue thromboplastin, tissues like the muscle were initially used to achieve hemostasis. Later, a wide range of chemicals were involved to activate the intrinsic and extrinsic coagulation systems. One of the first was the discovery that gelatin polymers could function as extrinsic thromboplastin, the chemical nature of gelatin is then modified in order to make it degradable by proteolysis, and the resultant material was absorbable gelatin, or "ab-gel."

Cellulose, a cell wall complex sugar, was also found to have tissue thromboplastin like activity, and this related to surgical cellulose, or surgicel [8], evolving as another locally acting styptic. Bovine and porcine pericardial collagen is a very strong extrinsic tissue thromboplastin, and this is used to buttress staple line, and data is now emerging that gastro intestinal staple lines that are buttressed with pericardium tend to bleed less. At this point of time, however, the cost is considerable and is a definite barrier to universal use. 
Further modification of the absorbable hemostat is the Surgicel, a product that is the fast acting absorbable cellulose, with bactericidal impregnation in order to decrease the incidence of clot infection that might follow the process of hemostasis.

Tissue Adhesives and Glues A number of chemical equations can help in changing the physical structure of tissues, and this can help in the surgical field and in the postoperative period. A range of chemicals are used for sealing and for adhesion, but a few will be discussed below:

1) Cyanoacrylate and butacrylate: These are used in the industry as superglues, and the principle of the glues is that these rapidly polymerize on exposure to the nitrogen of the atmosphere or when exposed to the moisture (of blood in the surgical fields).

The cyanoacrylate glue has been used to close the skin, after subcutaneous sutures, in situations where suture removal would be cumbersome. Foremost among this is the skin closure in the pediatric age group.

Butacrylate glue has been used extensively in tackling bleeding fundal varices in portal hypertension. As the polymerization and bonding time is in seconds, the fundal varix is injected and solidified very quickly. An important point to be remembered in the application of these glues is that the apparatus for delivery of the glues, for example, the endoscope and the injection sclerotherapy channel, could also become solidified, and therefore, normal saline is pushed in to flush out the glue simultaneously, otherwise it could have frustrating consequences for the surgeon and the endoscope as well!

2) Fibrin glues: A principle of these is to mix activated thrombin and fibrinogen to form an intense fibrinogen polymer (see Fig. 8). Useful in splenic, liver, and cardiac surgery, these fibrin glues are increasingly finding a number of other uses.

For example, a number of papers showed some advantage in using the fibrin glue to seal the subcutaneous tissue to the underlined structures and reduce the amount of fluid in the drain, and the fibrin glue is also gaining

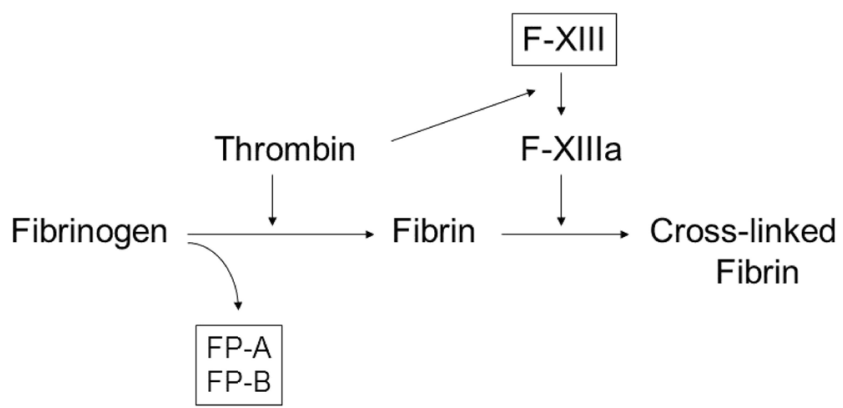

Fig. 8 Activated thrombin and fibrinogen forming an intense fibrinogen polymer acceptance as a sealant on staple lines, for example, after a sleeve gastrectomy or an intestinal anastomosis. Another attractive use of fibrin glue is to apply to fix the mesh while performing laparoscopic inguinal hernia meshplasty. Avoiding tackers or sutures is a boom for the surgeon [9].

3) Gelatin thrombin sealants: The very popular gelatin thrombin absorbable tissue sealants and hemostatic agents have the advantage that they can be evenly placed on delicate structures such as nerves.

4) Polyethylene glycol polymers (PEG): These are new sealants that have entered the market, and the advantage is the formation of a bio-adhesive and hemostatic bond, which then is absorbable over 6 weeks.

5) Amylopectin: This starch causes rapid contraction of the tunica media and causes an irritative closure of the endothelium. The authors have done a pilot trial on 24 patients, with excellent hemostatic results. The only problem with this chemical is that it is a starch, and when used in the abdominal cavity, it needs to be thoroughly washed off, for fear of long-term starch reaction, although nimal studies have not indicated any so for.

6) Albumin glutaraldehyde tissue adhesive: This expensive new adhesive is good for providing hemostasis in large vessels. It is used in endovascular repair of aortic aneurysms, etc., and pilot studies have shown it to be very helpful and dissecting out complex aneurysms with adhesion to the surrounding structure. The fear, with the usage of this chemical, is the possible development of a stricture or occlusion of vessels in relation the area of usage. Although it is still being evaluated, the initial studies are showing great promise.

Resistant infection in hospitals, wards, or operating theaters is literally a major bugbear for health care. An epoch making series of papers recently showed that the quaternary ammonium ion, when incorporated in sheets and various fabrics, could destroy most of the common bacteria that cause nosocornial infection, this new product, known as I-Shield or infection shield is all set to capture our practice in future. It is expected that surgical fabrics, gowns, and sheets made from this chemical will be safe to the human skin, while the same time being extremely lethal to micro organisms.

To conclude, this bird's eye view of the importance of understanding chemistry and chemical equation, both in the industrial surgical arena, as well as in the actual intraoperative scenario, is, admittedly, by no means complete. Such a qualification would result into one of gigantic proportions! However, the purpose here is to sensitize the readers to the magic of chemistry in our daily surgical life. One cannot but part with a quip, that chemistry between the patient and the surgeon still remains the most important type of chemistry! 


\section{References}

1. Guggenheim EA (1937) The theoretical basis of Raoult's law. Trans Faraday Soc 33:151-156. doi:10.1039/TF9373300151

2. Vecht J, Masclee AA, Lamers CB (1997) The dumping syndrome. Current insights into pathophysiology, diagnosis and treatment. Scand J Gastroenterol Supplement 223:21-27

3. Cockcroft DW, Gault MH (1976) Prediction of creatinine clearance from serum creatinine. Nephron 16:31-41. doi:10.1159/000180580

4. Lichtenstein IL, Shulman AG, Amid PK, Montllor MM (1989) The tension-free hernioplasty. Am J Surg 157(2):188-193
5. Gilbert AI, Graham MF, Voigt WJ (1999) A bilayer patch device for inguinal hernia repair, How To Do It, Hernia 3(3):161-166. First online: Springer

6. Robbins AW, Rutkow IM (1998) Mesh plug repair and groin hernia surgery. Surg Clin N Am 78(6):1007-1023

7. Usher FC (1962) Hernia repair with Marlex mesh: an analysis of 541 cases. Arch Surg 84:325-328

8. Levy ML, Day JD, Fukushima T, Batjer HH, Gamache FW Jr (1997) Surgicel fibrillar absorbable oxidized regenerated cellulose. Neurosurgery 41(3):701-702

9. Gibble JW, Ness PM (1990) Fibrin glue: the perfect operative sealant? Transfusion 30(8):741-747 